\title{
Personalized mechanical ventilation for acute respiratory distress syndrome: are we ready? - Maybe
}

\author{
Won-Young Kim ${ }^{1}$, Sang-Bum Hong ${ }^{2}$ \\ ${ }^{1}$ Department of Internal Medicine, Chung-Ang University Hospital, Chung-Ang University College of Medicine, Seoul, Korea; ${ }^{2}$ Departmemt of \\ Pulmonary and Critical Care Medicine, Asan Medical Center, University of Ulsan College of Medicine, Seoul, Korea \\ Correspondence to: Sang-Bum Hong, MD, PhD. Department of Pulmonary and Critical Care Medicine, Asan Medical Center, University of Ulsan \\ College of Medicine, 88 Olympic-ro 43-gil, Songpa-gu, Seoul 05505, Korea. Email: sbhong@amc.seoul.kr. \\ Provenance: This is an invited article commissioned by the Section Editor Xue-Zhong Xing [National Cancer Center (NCC)/Cancer Hospital, \\ Chinese Academy of Medical Sciences (CAMS) and Peking Union Medical College (PUMC), Beijing, China]. \\ Comment on: Constantin JM, Jabaudon M, Lefrant JY, et al. Personalised mechanical ventilation tailored to lung morphology versus low positive \\ end-expiratory pressure for patients with acute respiratory distress syndrome in France (the LIVE study): a multicentre, single-blind, randomised \\ controlled trial. Lancet Respir Med 2019;7:870-80.
}

Submitted Nov 27, 2019. Accepted for publication Dec 13, 2019.

doi: $10.21037 /$ jtd.2019.12.79

View this article at: http://dx.doi.org/10.21037/jtd.2019.12.79

Targeting specific biomarker-defined subgroups of heterogeneous syndromes, such as cancer or asthma, has fundamentally changed approaches to clinical management. For example, subphenotypes based on Th2type inflammation have been identified in asthma, leading to new targeted treatment approaches such as monoclonal antibodies against interleukin-13 (1). Acute respiratory distress syndrome (ARDS) is an acute, diffuse, inflammatory lung injury that is associated with a variety of etiologies and leads to severe respiratory failure refractory to conventional oxygen therapy (2). Lung protective ventilation strategies including low tidal volume and positive end-expiratory pressure (PEEP) are well-established treatments $(3,4)$, although numerous pharmacotherapies that seemed to show promise in preclinical studies have failed in clinical trials. ARDS is diagnosed based on clinical criteria rather than laboratory or pathophysiological criteria (5). ARDS cases may be very heterogenous with different responses to therapy. Calfee et al. identified two distinct ARDS subphenotypes in two randomized controlled trials (ARMA and ALVEOLI) $(3,4)$ using latent class analysis (6). Subphenotype 2 (a hyper-inflammatory ARDS) was associated with increased levels of inflammatory biomarkers, acidosis, shock, and mortality. In the ALVEOLI cohort, higher PEEP was beneficial in subphenotype 2 but harmful in subphenotype 1 . In subsequent studies, using latent class analysis in the FACTT and HARP-2 study populations $(7,8)$, the same group observed a survival benefit in the hyper-inflammatory subphenotype with conservative fluid management and simvastatin, respectively $(9,10)$. Interestingly, extrapulmonary factors seemed to contribute more to subphenotype classification than did pulmonaryspecific variables $\left(\mathrm{PaO}_{2} / \mathrm{FiO}_{2}\right.$ and ventilator parameters).

In a study that has now been described in Lancet Respiratory Medicine, Constantin et al. performed a multicenter, stratified, parallel-group, single-blind randomized controlled trial that included 20 intensive care units in France (11). According to the Berlin definition (5), adult patients with moderate to severe ARDS for less than $12 \mathrm{~h}$ were enrolled. Patients were randomly assigned to personalized group (ventilation strategy adjusted on the basis of lung morphology) or to control group (standard strategy). Lung morphology was assessed before randomization using a CT scan or chest $\mathrm{x}$-ray. Focal ARDS was defined as the presence of consolidations predominantly in the lower and back part of the lungs. Non-focal ARDS was defined as diffuse and patchy loss of aeration. Lung morphology was initially assessed by local site investigators, which then was re-assessed a posteriori by one radiologist and two intensivists. In the control group, patients received a tidal volume of $6 \mathrm{~mL} / \mathrm{kg}$, lower PEEP set using the $\mathrm{FiO}_{2}$-PEEP table proposed by ARDSNet (4), and prone 
positioning (12). In the personalized group, a tidal volume of $8 \mathrm{~mL} / \mathrm{kg}$, low PEEP, and prone positioning were applied in patients with focal ARDS. In patients with non-focal ARDS, a tidal volume of $6 \mathrm{~mL} / \mathrm{kg}$, recruitment maneuvers, and high PEEP were applied. The main finding was that personalized ventilation on the basis of lung morphology did not decrease 90-day mortality in contrast to classic low PEEP ventilation [hazard ratio (HR) 0.96; 95\% CI, 0.66-1.4; $\mathrm{P}=0.84]$. However, the lung morphology was misclassified for 85 (21\%) of 400 patients. When patients were analyzed after their lung morphology was correctly classified, a significant survival benefit was demonstrated in the personalized group [29 (19\%) of 156 patients vs. 58 (28\%) of 204 patients; $\mathrm{P}=0.042$ ]. The misclassified patients showed higher 90 -day mortality in the personalized group [26 $(65 \%)$ of 40 patients] than in the control group [18 (32\%) of 57 patients; $\mathrm{P}=0.012$ ].

These results demonstrate that personalized ventilation strategies might be beneficial in patients with ARDS. However, when personalized ventilation is incorrectly assessed, mortality increases considerably. Physiological studies have shown that low tidal volume, recruitment maneuvers, and higher PEEP may be more appropriate in non-focal ARDS, whereas higher tidal volume, lower PEEP, and prone positioning may be more beneficial in patients with focal ARDS $(13,14)$. In the ALVEOLI study (4), $\mathrm{FiO}_{2}$ and PEEP were adjusted without considering lung morphology, and PEEP did not affect patient survival. Conversely, low PEEP and prone positioning were applied in the intervention group of the PROSEVA trial (12), and the trial showed that prone positioning increases survival. In the recent ART study, recruitment maneuvers and high PEEP increased mortality in patients with ARDS (15). These inconsistent findings might be partly due to most trial designs focusing on a one-size-fits-all approach, without consideration of distinct responses to PEEP, recruitment maneuvers, and prone positioning. In the present study, at least one recruitment maneuver was performed to 82 (84\%) of 98 patients with non-focal ARDS in the personalized group. The average PEEP was 14 (SD 3) $\mathrm{cmH}_{2} \mathrm{O}$ in these patients compared with 10 (SD 2) $\mathrm{cmH}_{2} \mathrm{O}$ for those in the control group. Meanwhile, 92 (94\%) of 98 patients with focal ARDS in the personalized group had at least one session of prone positioning. In these patients, tidal volume was 7 (SD 1) $\mathrm{mL} / \mathrm{kg}$ and PEEP was 8 (SD 2) $\mathrm{cmH}_{2} \mathrm{O}$ compared with a tidal volume of 6 (SD 1) $\mathrm{mL} / \mathrm{kg}$ and PEEP of 11 (SD 2) $\mathrm{cmH}_{2} \mathrm{O}$ for patients in the control group. Fifty-two (25\%) of 204 patients in the control group had at least one session of prone positioning. As previously mentioned, a mortality reduction was observed when lung morphology and ventilation strategies were aligned.

Despite intriguing results, the present study has notable limitations. First, the number of patients with misclassified lung morphologies by the investigators was relatively high. This could be explained by the investigators' experience. With regard to diagnosis of ARDS based on lung morphology, agreement between the three experts was high $(\kappa=0.94)$. However, the agreement was moderate for local investigators $(\kappa=0.52)$. In a real-world setting, rapidly assessing lung morphology by local site investigators might not be feasible. In addition, the proportion of patients who had CT scans was low. CT scan was performed for $56(29 \%)$ of 196 patients in the personalized group and 80 (39\%) of 204 patients in the control group. Transportation of critically ill patients to obtain a CT scan can be difficult due to increased morbidity and mortality. Thus, it may be difficult to correctly phenotype patients at the bedside. To avoid misclassification in daily practice, alternative tools such as lung ultrasound $(16,17)$ and electrical impedance tomography $(18,19)$ can be safely used at the bedside. Like CT, bedside lung ultrasound allows a regional analysis of lung aeration and identifies ARDS lung morphology. It accurately assesses lung aeration changes following PEEP application (16) and prone positioning (17). Cinnella et al. applied electrical impedance tomography to assess the effects of recruitment maneuver (18). This strategy allowed a more homogeneous distribution of ventilation, and the increased ventilation to dorsal regions was correlated with arterial oxygen improvement. Second, this study was open label, and performance bias might have resulted in differences in the use of adjuvant treatments. In fact, only a quarter of patients were placed in the prone position at least once in the control group, although prone positioning was encouraged for all patients in this group. Third, mortality differences were significant between the correctly classified and incorrectly classified patients in the personalized group (Figure 2D). In the control group, however, misclassification of the focal and non-focal groups did not show any mortality difference (Figure 2C). Moreover, there was no difference in overall mortality between the personalized group and the control group [53 (27\%) of 196 patients vs. 56 (27\%) of 204 patients]. These findings suggest that personalized ventilation that is misaligned with lung morphology (e.g., open-lung ventilation in patients without alveolar recruitment) can be harmful, although a standard strategy (low tidal volume, low PEEP, and prone 
positioning) may not be harmful.

Together with findings from previous reports, the results presented by Constantin et al. (11) suggest that phenotypes of ARDS should be considered in clinical practice and for further studies. However, owing to study limitations, further studies are required. Correctly assigning the ventilation strategy for patients with ARDS requires rapid phenotyping, which can be challenging. The difficulty in correctly phenotyping patients at the bedside warrants further investigation.

\section{Acknowledgments}

None.

\section{Footnote}

Conflicts of Interest: The authors have no conflicts of interest to declare.

Ethical Statement: The authors are accountable for all aspects of the work in ensuring that questions related to the accuracy or integrity of any part of the work are appropriately investigated and resolved.

\section{References}

1. Corren J, Lemanske RF, Hanania NA, et al. Lebrikizumab treatment in adults with asthma. $\mathrm{N}$ Engl J Med 2011;365:1088-98.

2. Kim WY, Kang BJ, Chung CR, et al. Prone positioning before extracorporeal membrane oxygenation for severe acute respiratory distress syndrome: A retrospective multicenter study. Med Intensiva 2019;43:402-9.

3. Acute Respiratory Distress Syndrome Network, Brower RG, Matthay MA, et al. Ventilation with lower tidal volumes as compared with traditional tidal volumes for acute lung injury and the acute respiratory distress syndrome. N Engl J Med 2000;342:1301-8.

4. Brower RG, Lanken PN, MacIntyre N, et al. Higher versus lower positive end-expiratory pressures in patients with the acute respiratory distress syndrome. $\mathrm{N}$ Engl J Med 2004;351:327-36.

5. ARDS Definition Task Force, Ranieri VM, Rubenfeld GD, et al. Acute respiratory distress syndrome: the Berlin Definition. JAMA 2012;307:2526-33.

6. Calfee CS, Delucchi K, Parsons PE, et al. Subphenotypes in acute respiratory distress syndrome: latent class analysis of data from two randomised controlled trials. Lancet

Respir Med 2014;2:611-20.

7. National Heart, Lung, and Blood Institute Acute Respiratory Distress Syndrome Clinical Trials Network, Wiedemann HP, Wheeler AP, et al. Comparison of two fluid-management strategies in acute lung injury. $\mathrm{N}$ Engl J Med 2006;354:2564-75.

8. McAuley DF, Laffey JG, O'Kane CM, et al. Simvastatin in the acute respiratory distress syndrome. N Engl J Med 2014;371:1695-703.

9. Famous KR, Delucchi K, Ware LB, et al. Acute Respiratory Distress Syndrome Subphenotypes Respond Differently to Randomized Fluid Management Strategy. Am J Respir Crit Care Med 2017;195:331-8.

10. Calfee CS, Delucchi KL, Sinha P, et al. Acute respiratory distress syndrome subphenotypes and differential response to simvastatin: secondary analysis of a randomised controlled trial. Lancet Respir Med 2018;6:691-8.

11. Constantin JM, Jabaudon M, Lefrant JY, et al. Personalised mechanical ventilation tailored to lung morphology versus low positive end-expiratory pressure for patients with acute respiratory distress syndrome in France (the LIVE study): a multicentre, single-blind, randomised controlled trial. Lancet Respir Med 2019;7:870-80.

12. Guerin C, Reignier J, Richard JC, et al. Prone positioning in severe acute respiratory distress syndrome. $\mathrm{N} \mathrm{Engl} \mathrm{J}$ Med 2013;368:2159-68.

13. Puybasset L, Cluzel P, Gusman P, et al. Regional distribution of gas and tissue in acute respiratory distress syndrome. I. Consequences for lung morphology. CT Scan ARDS Study Group. Intensive Care Med 2000;26:857-69.

14. Gattinoni L, Caironi P, Cressoni M, et al. Lung recruitment in patients with the acute respiratory distress syndrome. N Engl J Med 2006;354:1775-86.

15. Writing Group for the Alveolar Recruitment for Acute Respiratory Distress Syndrome Trial Investigators, Cavalcanti AB, Suzumura EA, et al. Effect of Lung Recruitment and Titrated Positive End-Expiratory Pressure (PEEP) vs Low PEEP on Mortality in Patients With Acute Respiratory Distress Syndrome: A Randomized Clinical Trial. JAMA 2017;318:1335-45.

16. Bouhemad B, Brisson H, Le-Guen M, et al. Bedside ultrasound assessment of positive end-expiratory pressureinduced lung recruitment. Am J Respir Crit Care Med 2011;183:341-7.

17. Haddam M, Zieleskiewicz L, Perbet S, et al. Lung ultrasonography for assessment of oxygenation response to prone position ventilation in ARDS. Intensive Care Med 
2016;42:1546-56.

18. Cinnella G, Grasso S, Raimondo P, et al. Physiological Effects of the Open Lung Approach in Patients with Early, Mild, Diffuse Acute Respiratory Distress Syndrome: An Electrical Impedance Tomography Study. Anesthesiology
2015;123:1113-21.

19. Mauri T, Bellani G, Confalonieri A, et al. Topographic distribution of tidal ventilation in acute respiratory distress syndrome: effects of positive end-expiratory pressure and pressure support. Crit Care Med 2013;41:1664-73.

Cite this article as: Kim WY, Hong SB. Personalized mechanical ventilation for acute respiratory distress syndrome: are we ready? —Maybe. J Thorac Dis 2019;11(12):5658-5661. doi: $10.21037 /$ jtd.2019.12.79 